\title{
Analysis of aflatoxins in rustically wines from eastern Romania using the direct real time method (DART)
}

\author{
A.-M. Nistor ${ }^{1}$, Ş.-D. Cotan $^{1}$, V.V. Cotea ${ }^{1,2}$, and M. Niculaua ${ }^{2}$ \\ 1 "Ion Ionescu de la Brad" University of Agricultural Sciences and Veterinary Medicine, 3 Mihail Sadoveanu Alley, \\ 700490 Iaşi, Romania \\ ${ }^{2}$ Research Centre for Oenology, Romanian Academy Iasi Branch, 9 Mihail Sadoveanu Alley, 700490 Iaşi, Romania
}

\begin{abstract}
Romania has a long tradition regarding the production of wine rustically or household products. Each householder has the possibility for making its own wine from its own grape vines. Most of them are made and kept using almost no interventions or treatments no corrections or added substances, which could protect them from eventual diseases, which may appear during the winemaking process. The grapes are collected manually by each family alone and it can be called is itself a ritual specific to the each area and a symbol of autumn. In this context, several samples of wine were collected from households located in five Romania eastern counties (Iaşi, Vaslui, Galaţi, Brăila, Ialomiţa and Tulcea). The samples were neither filtered, nor treated against the developing secondary fermentations. This fact increases the risk of infestation with mycotoxins. Twenty samples belonging to the aforementioned regions were analysed using the direct real time method (DART). This method is very fast and does not require any prior preparation of the samples. The goal was to identify the aflatoxins B1, B2, G1 and G2 that are known to be frequently present in wines. From the total of the analysed samples, in four of them it was detected a concentration greater than $20 \mu \mathrm{g} / \mathrm{kg}$.
\end{abstract}

\section{Introduction}

Romania is like a vine leaf for some people because of its shape and is one of the most important viticultural European countries. According to the 2017 OIV report, Romania is the $10^{\text {th }}$ country world-wide regarding its vine surface [1]. There are historical testimonials, which prove that the wild vine Vitis silvetis was cultivated in this region some around 7000 B.C. The tools used in those times for viticulture are still kept as testimonials in history museums around the country. Nowadays, viticultural plantations are spread in the whole country, several geographic areas with different production technics being thus visible.

The wine has always had a special meaning for religious rituals (baptisms, weddings, funerals etc.) still maintained nowadays. Grape harvest is one of the most important events during autumn. This moment was a period for family reunions, forming new friendships and spending time together in small and large communities alike. It was said that during that time the entire village's problems were put on hold, the schools were on break and all life was taking place in the vineyards $[2,3]$.

Some traditions are kept even today, especially in the rural countryside. In Romania, almost each household is proud of its own wine. People are very fond of household wine, which is made from one's own sources. However, even if the trends have evolved and technology has developed, a series of problems with these practices remains. Most of these grapes are not scientifically processed and therefore are not protected against microbial development or secondary fermentations. Another problem that arises in this context is the lack of qualification of the personnel that is in charge of harvesting the grapes (these being members of the family or a small community). Also, the lack of their knowledge regarding the factors, which can affect grape health and wine respectively: improper sorting of damaged grapes from healthy ones, lack of clean transportation means and storage and strict hygiene comparing with an industrial winery's standards.

All these facts set off an alarm and laid the foundations of this study. Samples of household wine were collected from several regions in Romania and the level of aflatoxins (substances that can appear in wine following the development of mould on grapes) contamination was monitored.

There are numerous studies, which indicate that the geographical position has an influence on the incidence of mycotoxin development. Samples coming from areas with different latitudes showed the fact that in southern Europe the incidence of mycotoxin contamination is higher than in northern Europe. The main reason is the climate - the colder and less humid weather in the northern part compared with the higher temperatures and the cycles of drought and humidity in the southern part, which contribute to the stress, and degradation of the wines [4-6].

Aflatoxins are extremely toxic chemical compounds, especially the B1 aflatoxin that is classified by IARC as being carcinogenic [7]. They can easily develop on grapes when the culture is affected by mould and can be passed on to the wine because aflatoxins are stable chemical compounds. 
Table 2. Calibration curve characteristics.

\begin{tabular}{|c|c|c|c|}
\hline Aflatoxins & a & b & $\mathbf{R}^{2}$ \\
\hline AFB1 & 0.00002 & 0.4871 & 0.9179 \\
\hline AFB2 & 0.0000007 & 0.9513 & 0.9172 \\
\hline AFG1 & 0.00005 & -2.199 & 0.9817 \\
\hline AFG2 & 0.000008 & 1.5781 & 0.9893 \\
\hline
\end{tabular}

is used to generate $\mathrm{M}+\mathrm{H}^{+}$ions and, for sampling, an automatic micropipette $0.5-10 \mu \mathrm{L}$ (Hirschmann Laborgerate) was employed. For preparing the stock solutions, a $50 \mu \mathrm{L}$ syringe with cemented needle PT2 universal type (Hamilton ${ }^{\mathrm{TM}}$ ) was used in order to extract standard solution through the septum without any risks of contamination.

Table 1. Sample (S) number and corresponding county (C).

\begin{tabular}{|c|c|c|c|c|c|}
\hline $\mathbf{S}$ & $\mathbf{C}$ & $\mathbf{S}$ & $\mathbf{C}$ & $\mathbf{S}$ & $\mathbf{C}$ \\
\hline 1 & BR & 7 & $\mathrm{VS}$ & 13 & TL \\
\hline 2 & BR & 8 & VS & 14 & IL \\
\hline 3 & BR & 9 & VS & 15 & IL \\
\hline 4 & BR & 10 & VS & 16 & GL \\
\hline 5 & VS & 11 & VS & 17 & GL \\
\hline 6 & VS & 12 & IS & 18 & IS \\
\hline & & & & 19 & IS \\
\hline
\end{tabular}

\section{Experimental}

The samples were analysed using real time direct analysis (DART). This technique has the advantages that it is very fast, reproducible and does not require any special preparation for the samples. Different kinds of analysis for liquids or solid samples can be realised with this technic. The analysis is performed at atmospheric pressure and involves a helium gas-phase ionization mechanism. The coupling with the mass spectrometer allows identifying compounds of interest.

\subsection{Wine samples}

The wine samples come from different vineyards from six Romanian counties, located from north to south in this order: Iaşi, Vaslui, Galaţi, Brăila, Tulcea and Ialomiţa. The most northern location from which a sample was selected had a latitude of $46^{\circ} 26^{\prime} \mathrm{N}$ and the most southern had a latitude of $44^{\circ} 38^{\prime} \mathrm{N}$, as shown in Fig. 1 .

The wines were randomly selected from households based for a larger spatial distribution. There are 20 samples, that come from the 2017 harvest with both white and red grapes. None of the samples suffered any conditioning treatments during the wine making process. Out of the total number of samples (S), three were from Iaşi (IS), seven from Vaslui (VS), two from Galaţi (GL), four from Brăila (BR), one from Tulcea (TL) and two from Ialomiţa (IL) (Table 1).

\subsection{Chemicals and materials}

The mycotoxin standard is a mixture from B1 aflatoxin (AFB1), B2 aflatoxin (AFB2), G1 aflatoxin (AFG1) and $\mathrm{G} 2$ aflatoxin (AFG2), with the following concentrations: $2 \mu \mathrm{g} / \mathrm{mL}$ for AFB 1 and AFG1, $0.503 \mu \mathrm{g} / \mathrm{mL}$ for AFB2 and 0.504 for AFG2, all in acetonitrile. The mixture was from LGC Standards Germany.

The calibration curve is made with $99.9 \%$ purity acetonitrile $\left(\mathrm{Merck}^{\mathrm{TM}}\right)$; $98-100 \%$ formic acid $\left(\mathrm{Merck}^{\mathrm{TM}}\right)$

\subsection{Preparation of stock solutions and wine samples}

The stock solutions were prepared by diluting with 1:2 acetonitrile from the original standard mix, obtaining the first level of the calibration curve. The next level was obtained by diluting the resulting solution at $1: 2$ and the last level by diluting at $1: 10$. Therefore, the calibration curve was built on linear model with three concentration levels, each level having three repetitions. $20 \mu \mathrm{L}$ were pipetted on the sample's stand for each calibration point. The obtained values for the equation are presented in the Table 2 and fit well the linear model:

The same method in which $10 \mu \mathrm{L}$ of wine with and without $10 \mu \mathrm{L}$ of the stock solution with the aflatoxin mix were pipetted (spiked solutions) were analysed in triplicate. It is worth mentioning that the wine samples showed superficially high tension on the stainless steel mesh and the waiting time for drying each sample was between 90-120 minutes. The drying of the sample was performed only in open atmosphere at room temperature in order to not degrade the aflatoxins.

For the calibration curve as well as for the rest of the samples, the ionization could not be realised without adding an ionization agent, $10 \mu \mathrm{L}$ of acetonitrile with $1 \%$ formic acid respectively.

\subsection{DART-orbitrapMS}

The analysis is conducted on a high resolution mass spectrometer (HRMS) Thermo Scientific Q Exactive with an atmospheric pressure interface (API) Interface Vapur from IonSence known as DART (Direct analysis in real time) with transmission module at $350^{\circ} \mathrm{C}, 3.5 \mathrm{KV}$, positive mode with helium for ionization placed at $35 \mathrm{~mm}$ from the interface sublimation grid. The speed for analysis in the ionisation interface is $0.5 \mathrm{~mm} / \mathrm{s}$ and all samples are done in triplicate and spiked. The standby gas used is nitrogen at $40 \mathrm{psi}$; the same pressure as in analysis mode. The DART grid is a stainless steel $74 \times 74$ mesh at which the samples and standards are spotted to dryness. In front of the HRMS, there is a Vapur interface that reduce the amount of molecules and quasi-ions that enter the ion path. The mass spectrometer is controlled via Tune software ver. 2.9 and the integration and processing of data is made in an Xcalibur software ver. 3.0. Targeted quantification by single ion monitoring (t-SIM) mod was utilised at 140,000 FWHM for the following masses: AFB1 $\left(\mathrm{C}_{17} \mathrm{H}_{1} 2 \mathrm{O}_{6}\right)$ 
Table 3. Concentrations for aflatoxins in $\mathrm{ppb}$.

\begin{tabular}{|c|c|c|c|c|}
\hline Sample & AFB1 \pm SD $(\mathbf{p p b})$ & AFB2 \pm SD $(\mathbf{p p b})$ & AFG1 \pm SD $(\mathbf{p p b})$ & AFG2 \pm SD $(\mathbf{p p b )}$ \\
\hline 1 & $12.30 \pm 1.26$ & $1.21 \pm 0.25$ & $4.84 \pm 0.41$ & $1.60 \pm 0.19$ \\
\hline 2 & $19.64 \pm 3.4$ & $2.94 \pm 0.28$ & $9.15 \pm 1.03$ & $2.00 \pm 0.34$ \\
\hline 3 & $\mathrm{ND}$ & $\mathrm{ND}$ & $\mathrm{ND}$ & $5.79 \pm 0.67$ \\
\hline 4 & $\mathrm{ND}$ & $\mathrm{ND}$ & $22.84 \pm 2.34$ & $5.04 \pm 0.54$ \\
\hline 5 & $14.90 \pm 1.78$ & $1.85 \pm 0.31$ & $\mathrm{ND}$ & $\mathrm{ND}$ \\
\hline 6 & $19.74 \pm 2.57$ & $3.74 \pm 0.46$ & $15.40 \pm 2.06$ & $4.72 \pm 0.34$ \\
\hline 7 & $16.55 \pm 3.23$ & $1.22 \pm 0.36$ & $12.86 \pm 1.45$ & $5.09 \pm 0.56$ \\
\hline 8 & $\mathrm{ND}$ & $\mathrm{ND}$ & $\mathrm{ND}$ & $5.67 \pm 0.99$ \\
\hline 9 & $19.16 \pm 3.67$ & $1.94 \pm 0.32$ & $14.81 \pm 1.78$ & $5.29 \pm 0.71$ \\
\hline 10 & $12.12 \pm 1.98$ & $0.24 \pm 0.06$ & $11.25 \pm 1.24$ & $3.21 \pm 0.35$ \\
\hline 11 & $18.61 \pm 2.08$ & $3.55 \pm 0.35$ & $19.74 \pm 2.34$ & $4.72 \pm 0.32$ \\
\hline 12 & $\mathrm{ND}$ & $2.65 \pm 0.25$ & $\mathrm{ND}$ & $\mathrm{ND}$ \\
\hline 13 & $17.48 \pm 4.87$ & $2.42 \pm 0.17$ & $18.33 \pm 1.98$ & $7.09 \pm 1.03$ \\
\hline 14 & $31.71 \pm 5.78$ & $\mathrm{ND}$ & $\mathrm{ND}$ & $6.51 \pm 0.98$ \\
\hline 15 & $32.27 \pm 6.74$ & $\mathrm{ND}$ & $26.68 \pm 5.21$ & $5.53 \pm 0.89$ \\
\hline 16 & $19.28 \pm 2.67$ & $1.33 \pm 0.14$ & $7.06 \pm 0.82$ & $1.33 \pm 0.24$ \\
\hline 17 & $\mathrm{ND}$ & $\mathrm{ND}$ & $\mathrm{ND}$ & $\mathrm{ND}$ \\
\hline 18 & $\mathrm{ND}$ & $\mathrm{ND}$ & $\mathrm{ND}$ & $\mathrm{ND}$ \\
\hline 19 & $33.68 \pm 6.21$ & $3.54 \pm 0.65$ & $\mathrm{ND}$ & $\mathrm{ND}$ \\
\hline
\end{tabular}

$\mathrm{M}=313.07066 \mathrm{Da} ;$ AFB2 $\left(\mathrm{C}_{17} \mathrm{H}_{1} 4 \mathrm{O}_{6}\right) 315.08631 \mathrm{Da}$; $\operatorname{AFG} 1\left(\mathrm{C}_{17} \mathrm{H}_{1} 2 \mathrm{O}_{7}\right) \mathrm{M}=329.06558 \mathrm{Da} ; \mathrm{AFG} 2\left(\mathrm{C}_{17} \mathrm{H}_{1} 4 \mathrm{O}_{7}\right)$ $\mathrm{M}=331.08123 \mathrm{Da}$. The mono-isotopic species are eliminated from calculation and only the protonated species are used in the quantification after processing with standard peak algorithm Avalon and 15 points Gaussian smoothing. The accumulation time for the experiment is set to $100 \mathrm{~ms}$ with an isolation window of $4 \mathrm{Da}$. Parallel to this, a full scan from $50-375 \mathrm{Da}$ is conducted with the same MS condition for structural elucidation and background evaluation.

\section{Results and discussion}

The results show that $\mathrm{B} 1$ and $\mathrm{G} 1$ aflatoxins are present in higher concentrations in the samples and B2 and $\mathrm{G} 2$ in smaller concentrations. This is due to the fact that AFB1 is the most widespread out of all four compounds (Table 3).

The impact of the sample amount is one of the reason that we can characterise wine samples directly with the DART method. On the other hand, the amount of time that is lost for drying the wine droplet is ideal for mobile sampling with the possibility for later analysis.

All of the four analysed aflatoxins are present in almost half of all the samples. There are some samples, that only contain one class of aflatoxin - samples 5 and 19 contain only AFB1 and AFB2 and sample 4 contains only AFG1 and AFG2. Samples 3 and 8 contain only AFG2 and sample 12 only AFB2. The last two aforementioned samples have a low total aflatoxin level and their values are very small. There are, however, samples in which the concentration was below the method's detectability threshold, namely samples 17 and 18 . These two samples come from Iaşi and Galaţi counties and the lack of aflatoxins is probably due to a cleaner winemaking process or due to healthier grapes, given the fact that northern areas have better climate.

The highest concentrations obtained is for AFB1 and AFG1, that go above $20 \mathrm{ppb}$, are between $26.68 \mathrm{ppb}$ and $33.68 \mathrm{ppb}$. Many of the samples have values close to $20 \mathrm{ppb}$, considered unofficially the threshold for healthy products. It is observed that three out of the four samples with the highest values come from Ialomiţa county, which is the most southern out of all the targeted counties. Sample 15 seems to be the most affected, having the highest levels of AFB1 and AFG1.

One can register also that the AFB1 level is found in three out of our samples, compared to the AFG1 level. When one of them is present in a sample in a high enough concentration, the other has a similar value.

\section{Conclusions}

The chosen method was fast to detect and quantify, highly sensitive and allowed identifying the aflatoxins down to $\mathrm{ppb}$ levels of concentration. The standard deviation (SD) was between $13-25 \%$.

The aflatoxin level found in the analysed samples was not present in a series of samples and over $20 \mathrm{ppb}$ in three cases with a maximum of $33.68 \mathrm{ppb}$ AFB1 for one sample in the northern part of Romania.

The samples have scattered values in the proposed spatial distribution.

For future references, traditional winemaking practices should be improved regarding hygienic conditions and grape selection, while also using correct stabilisation treatment in order to decrease the risk of aflatoxin incurrence.

The authors would like to thank the Institute of Macromolecular Chemistry "Petru Poni" Iaşi for supplying the standard solutions used in this study and to the "V. Adamachi" Didactic Farm from "Ion Ionescu de la Brad" University of Agricultural Sciences and Veterinary Medicine for their involvement in procuring the samples. Special thanks to laboratory colleagues Dr. Ioan Moraru and Cristina Scutăraşu for their help in preparing the samples.

\section{References}

[1] OIV Statistical Report on World Vitiviniculture, World VitiViniculture Situation, 5 (2017)

[2] A. Mihalca, În onoarea viei şi vinului, 25 (2008)

[3] V.V. Cotea, F. Andreescu, Romania - The Land of Wine 11 (2008) 
[4] J. Gil-Serna, C. Vázquez, M.T. González-Jaén, B. Patiño, A Rev. Beverages 4, 1 (2018)

[5] I. Stefanaki, E. Foufa, A. Tsatsou-Dritsa, P. Dais, Phytopat. Medit. 51, 131 (2012)

[6] S. Rotaru, F. Israel-Roming, G. Campeanu, G. Deciu, Ro. Biotech. Lett. 16, 126 (2011)
[7] IARC, http://monographs.iarc.fr/ ENG/Classification/ (2016)

[8] M. Tamura, A. Uyama, N. Mochizuki, Analyt. Sc. 27, 629 (2011)

[9] L. Vaclavik, M. Zachariasova, V. Hrbek, J. Hajslova, Talanta 82, 1950 (2010) 